\title{
De Sitter stability in quadratic gravity
}

\author{
A. V. Toporensky ${ }^{\dagger}$ and P. V. Tretyakov ${ }^{\ddagger}$ \\ Sternberg Astronomical Institute, Universitetsky prospect, 13, Moscow \\ 119992, Russia \\ ¥ Electronic mail: tpv@xray.sai.msu.ru \\ $\dagger$ Electronic mail: lesha@sai.msu.ru
}

\begin{abstract}
Quadratic curvature corrections to Einstein-Hilbert action lead in general to higher-order equations of motion, which can induced instability of some unperturbed solutions of General Relativity. We study conditions for stability of de Sitter cosmological solution. We argue that simple form of this condition known for FRW background in $3+1$ dimensions changes seriously if at least one of these two assumptions is violated. In the present paper the stability conditions for de Sitter solution have been found for multidimensional FRW background and for Bianchi I metrics in $3+1$ dimensions.
\end{abstract}

\section{Introduction}

Recently theories of higher-order gravity have become a matter of intensive investigations. Some of them are motivated by string theory [1], other proposals have been put forward in order to explain present accelerated expansion of the Universe (see [2, 3] and references therein). One of the main features of these theories is the fact that they generally lead to equations of motion which contain higher-order derivatives. In $3+1$ dimensions all higher-curvature terms in Lagrangian which keep the equations of motion to be the same order differential equations as in General Relativity vanish [4]. In string gravity the second order correction contains dilaton scalar field in addition to the gravity sector. These corrections has a particular structure (a product of Gauss - Bonnet term and a function of a dilaton field) which prevents the resulting equations of motion from being of higher-order equation. However, these equations become higherderivative starting from the $3-\mathrm{d}$ order corrections (see, for example, [5].)

As the number of time derivatives in the equations of motions increases in comparison with the situation in classical General relativity, the dimensionality of the phase space of the corresponding dynamical system increases as well. This may result in a dynamical behavior qualitatively different from that known 
in GR: some important GR solutions can loose their stability (this effect from a general point of view have been described in [6]). Though higher-derivative terms may be small on classical solution, perturbations from higher-order corrections can grow in direction of "additional" dimensions of the phase space, and a point which is stable in GR may become unstable in higher-order theory. This situation has already been described in 4-th order string gravity for Schwarzschild [7] and de Sitter solutions [8], as well as in cosmological scenarios with vacuum polarization [9]. The other proposal of this type is so called modified gravity with corrections in the form of function of the curvature $f(R)$ 10, 11] which is also not free from instabilities [12, 13] (see, however, [14] and recent rewiews [15, 16]).

Recent understanding that modern string theories may admit many (actually, very many) solutions in which 4 -D part is the (metastable) de Sitter space-time with a positive effective cosmological constant ([17, 18] and many following papers) renew interest in the investigation of the stability of de Sitter solutions in gravity theories containing higher order powers of the Riemann tensor in their effective Lagrangian. These terms were long known to arise from quantum-gravitational corrections to the Einstein gravity (either the vacuum one, or interacting with matter quantum fields). Presumably, some of them may remain in the string theory in spite of many cancellations due to its numerous symmetries. Many particular forms of second order curvature terms have been studied in connection to possible instabilities induced by them [19, 20, 21]. This work have been also extended to $R^{n}$ gravity [22, 23]. In the present paper we study stability of de Sitter solution with respect to second order curvature corrections in their general form.

\section{FRW Universe in $3+1$ dimension}

A general form of second order curvature corrections is

$$
S_{2}=\int \sqrt{-\mathrm{g}}\left(\alpha R^{i k l m} R_{i k l m}+\beta R^{i k} R_{i k}+\gamma R^{2}\right) d^{4} x .
$$

However, in $3+1$ dimensions the Gauss-Bonnet term

$$
G B=R^{i k l m} R_{i k l m}-4 R^{i k} R_{i k}+R^{2}
$$

does not contribute to equations of motion, and, then, we can rewrite (1) in the form

$$
S_{2}=\int \sqrt{-\mathrm{g}}\left(\alpha R^{i k l m} R_{i k l m}+\beta R^{i k} R_{i k}+\gamma R^{2}-\alpha G B\right) d^{4} x .
$$

Introducing $B=\beta+4 \alpha, C=\gamma-\alpha$ we get the following action for the theory with a cosmological constant term

$$
S=\int \sqrt{-\mathrm{g}}\left(R+B R^{i k} R_{i k}+C R^{2}-\Lambda\right) d^{4} x .
$$


For the Friedmann-Robertson-Walker metrics

$$
\mathrm{g}_{i k}=\operatorname{diag}\left(-n(t)^{2}, a(t)^{2}, a(t)^{2}, a(t)^{2}\right) .
$$

we have

$$
\begin{gathered}
R=\frac{6}{n^{2}}\left[\frac{\ddot{a}}{a}-\frac{\dot{a}}{a} \frac{\dot{n}}{n}+\frac{\dot{a}^{2}}{a^{2}}\right], \\
R_{i k}^{2}=\frac{12}{n^{4}}\left[\frac{\ddot{a}^{2}}{a^{2}}-\frac{\ddot{a}}{a} \frac{\dot{a}}{a} \frac{\dot{n}}{n}+\frac{\dot{n}^{2}}{n^{2}} \frac{\dot{a}^{2}}{a^{2}}+\frac{\ddot{a}}{a} \frac{\dot{a}^{2}}{a^{2}}-\frac{\dot{a}^{3}}{a^{3}} \frac{\dot{n}}{n}+\frac{\dot{a}^{4}}{a^{4}}\right] .
\end{gathered}
$$

Varying the action (3) with respect to $n$ and setting $n=1$ we obtain the following analog of Friedmann equation (we denote $D=12 B+36 C$ ):

$6 H^{2}-\Lambda+2 D H^{2}\left(\dot{H}+H^{2}\right)-3 D H^{4}-D\left(\dot{H}+H^{2}\right)^{2}+2 D H\left(\ddot{H}+3 H \dot{H}+H^{3}\right)=0$,

where $H \equiv \frac{\dot{a}(t)}{a(t)}$ is the Hubble parameter.

Note that the Friedmann equations, being algebraic in GR, becomes a differential one in the theory with second order curvature corrections when $D \neq 0$. In the $D=0$ case the Friedmann equation does not change at all, and we exclude this case from the further analysis.

For stability studies this equation should be written in the form of a system of two first order equations:

$$
\begin{aligned}
& \dot{H}=F, \\
& \dot{F}=-3 H F-H^{3}+\frac{1}{2 D H}\left(\Lambda-6 H^{2}+2 D H^{4}+D F^{2}\right) \equiv f .
\end{aligned}
$$

The form of de Sitter solution $(\dot{H}=0, \dot{F}=0)$ give us the equations for corresponding stable points:

$$
-H_{0}^{3}+\frac{1}{2 D H_{0}}\left(\Lambda-6 H_{0}^{2}+2 D H_{0}^{4}\right)=0
$$

its solution is $H_{0}= \pm \sqrt{\frac{\Lambda}{6}}$. It is remarkable, that this de Sitter solution is exactly the same as in the pure GR, so higher-order corrections in the theory under investigation do not shift the location of the fixed point. However, its stability can be affected by the higher-order terms.

Linearizing (6) we obtain

$$
\begin{aligned}
& \dot{H}=F \\
& \dot{F}=\left(\frac{\partial f}{\partial F}\right)_{0} F+\left(\frac{\partial f}{\partial H}\right)_{0} H,
\end{aligned}
$$

where

$$
\begin{gathered}
\frac{\partial f}{\partial F}=-3 H+\frac{F}{H} \\
\frac{\partial f}{\partial H}=-3 F-3 H^{2}-\frac{\Lambda-6 H^{2}+2 D H^{4}+D F^{2}}{2 D H^{2}}+\frac{-12 H+8 D H^{3}}{2 D H} .
\end{gathered}
$$


Two eigenvalues of this system are

$$
\mu_{1,2}=\frac{1}{2}\left[\left(\frac{\partial f}{\partial F}\right)_{0} \pm \sqrt{\left(\frac{\partial f}{\partial F}\right)_{0}^{2}+4\left(\frac{\partial f}{\partial H}\right)_{0}}\right] .
$$

For stability of the solution it is necessary that both eigenvalues have negative real parts. Substituting the de Sitter solution we get that in a stable point $\left(\frac{\partial f}{\partial F}\right)_{0}=-3 H_{0}$.

As $\left(\frac{\partial f}{\partial F}\right)_{0}<0$ in an expanding Universe, negativity of eigenvalues requires $\left(\frac{\partial f}{\partial H}\right)_{0}<0$. On the other hand, we have from (7) that in the de Sitter point

$$
\left(\frac{\partial f}{\partial H}\right)_{0}=\frac{-6}{D}
$$

which means that the condition for stability of the de Sitter solution is $D=$ $12(\alpha+\beta+3 \gamma)>0$.

In is possible to consider this result from a different point of view. Instead of Riemann tensor, we can express second order corrections through Weil tensor

$$
C_{i k l m}^{2}=R_{i k l m}^{2}+(N-6) R_{i k}^{2}+\left(\frac{7}{3}-\frac{13}{18} N+\frac{1}{18} N^{2}\right) R^{2},
$$

where $N$ is the dimensionality of space-time and the curvature scalar:

$$
S=\int \sqrt{-\mathrm{g}}\left(R+\tilde{B} C^{i k l m} C_{i k l m}+\tilde{C} R^{2}-\Lambda\right) d^{4} x,
$$

where $\tilde{B}=2 B=2 \alpha+\frac{1}{2} \beta$ and

$$
\tilde{C}=\frac{1}{3}(B+3 C)=\frac{1}{3}(\alpha+\beta+3 \gamma)=D / 36 \text {. }
$$

The Weil tensor vanishes on the Friedmann metrics, and the general quadratic corrections reduce to the $R^{2}$-term with the known result: the de Sitter solution is stable if the coefficient before $R^{2}$-term in (8) is positive.

\section{Multidimensional FRW Universe}

It is instructive to compare this result with the situation in higher dimensional theory, where the Gauss-Bonnet term does contribute to dynamical equations. To illustrate importance of this property, we consider the theory with second order curvature corrections, which we write down in the form

$$
S=\int \sqrt{-\mathrm{g}}\left(R+A C^{i k l m} C_{i k l m}+B R^{i k} R_{i k}+C R^{2}-\Lambda\right) d^{N} x,
$$

As usual, $C_{i k l m}=0$ on the Friedmann metrics, and we have two independent parameters of the theory $B$ and $C$. The explicit forms of second order corrections on the Friedmann metrics are 


$$
\begin{gathered}
R=\frac{(N-1)}{n^{2}}\left[2 \frac{\ddot{a}}{a}-2 \frac{\dot{n}}{n} \frac{\dot{a}}{a}+(N-2) \frac{\dot{a}^{2}}{a^{2}}\right] \\
R_{i k}^{2}=\frac{(N-1)}{n^{4}}\left[N \frac{\ddot{a}^{2}}{a^{2}}-2 N \frac{\dot{n}}{n} \frac{\dot{a}}{a} \frac{\ddot{a}}{a}+N \frac{\dot{n}^{2}}{n^{2}} \frac{\dot{a}^{2}}{a^{2}}+2(N-2) \frac{\ddot{a}}{a} \frac{\dot{a}^{2}}{a^{2}}-2(N-2) \frac{\dot{n}}{n} \frac{\dot{a}^{3}}{a^{3}}+(N-2)^{2} \frac{\dot{a}^{4}}{a^{4}}\right] \\
R_{i k l m}^{2}=\frac{2(N-1)}{n^{4}}\left[2 \frac{\ddot{a}^{2}}{a^{2}}-4 \frac{\dot{n}}{n} \frac{\dot{a}}{a} \frac{\ddot{a}}{a}+2 \frac{\dot{n}^{2}}{n^{2}} \frac{\dot{a}^{2}}{a^{2}}+(N-2) \frac{\dot{a}^{4}}{a^{4}}\right]
\end{gathered}
$$

Varying (9) with respect to $n$, setting $n=1$ and solving the equation of motion with respect to the second derivative we get

$$
\begin{aligned}
& \ddot{H}=\frac{-1}{2 H(N-1)[B N+4 C(N-1)]}\left[(N-1)(N-2) H^{2}-\Lambda+B\left\{(N-1)^{2}(N-4) H^{4}\right.\right. \\
& \left.-N(N-1) \dot{H}^{2}+2 N(N-1)^{2} \dot{H} H^{2}\right\}+C\left\{N(N-1)^{2}(N-4) H^{4}-4(N-1)^{2} \dot{H}^{2}\right. \\
& \left.\left.+8(N-1)^{3} \dot{H} H^{2}\right\}\right] \equiv f .
\end{aligned}
$$

It should be noted that if $B N+4 C(N-1)=0$ the resulting equation of motion does not contain $\dot{H}$ and $\ddot{H}$ terms, which means that a new de Sitter solution is trivially stable. This case should be excluded from our analysis.

The de Sitter fixed points can be found from the equation

$$
(N-1)^{2}(N-4)(B+C N) H_{0}^{4}+(N-1)(N-2) H_{0}^{2}-\Lambda=0,
$$

and have the form

$$
H_{0}^{2}=\frac{-(N-2) \pm \sqrt{(N-2)^{2}+4 \Lambda(N-4)(B+C N)}}{2(N-1)(N-4)(B+C N)}
$$

Comparing these results with those obtained in Sec.2 we can see two important differences from the previously studied case of $3+1$ dimensions:

- The de Sitter points are shifted in comparison with classical point $H^{2}=$ $\Lambda /((N-1)(N-2))$. It happens due to the first term in eq.(14) which vanishes in $N=4$ dimensions.

- There are two different de Sitter solutions, corresponding to two positive roots of (15). Each of them has its own zone of existence, so depending on $B$ and $C$ we can have two or one de sitter solutions, or the situation when de Sitter solutions are absent.

The point with (+) sign in (15) has a regular behavior for $B \rightarrow 0, C \rightarrow 0$ (it tends to unperturbed de Sitter solution), the behavior of the point with (-) sign is singular. The latter point can not be an analog of any de Sitter point, existing in the pure GR, and exists even in the case of $\Lambda=0$. 
For stability analysis we should rewrite (13) in the form of dynamical system (7). Using the same notations as in the previous section, we get

$$
\left(\frac{\partial f}{\partial F}\right)_{0}=-(N-1) H_{0}
$$

and, for the same reason, the condition for de Sitter stability is equivalent to negativity of $\left(\frac{\partial f}{\partial H}\right)_{0}$. The eq.(13) gives

$$
\begin{aligned}
& \left(\frac{\partial f}{\partial H}\right)=-\frac{f}{H}-\frac{1}{2 H(N-1)[B N+4 C(N-1)]}\left[2(N-1)(N-2) H+B\left\{4(N-1)^{2}(N-4) H^{3}\right.\right. \\
& \left.\left.+4 N(N-1)^{2} \dot{H} H\right\}+C\left\{4 N(N-1)^{2}(N-4) H^{3}+16(N-1)^{3} \dot{H} H\right\}\right] .
\end{aligned}
$$

In the de Sitter point we get

$$
\left(\frac{\partial f}{\partial H}\right)_{0}=\mp \frac{\sqrt{(N-2)^{2}+4 \Lambda(N-4)(B+C N)}}{B N+4 C(N-1)},
$$

We get that stability conditions depend on the sign of the combination $E=$ $B N+4 C(N-1)$. If it is positive, the regular de Sitter point (if exists) is always stable, while the singular de Sitter point is always unstable, independently of $\Lambda$. If it is negative, the regular point is unstable, and the singular point is stable.

The conditions for existence of these de Sitter points can be easily derived from (15). Two conditions which should be satisfied are: $(N-2)^{2}+4 \Lambda(N-$ $4)(B+C N)>0$ and $H_{0}^{2}>0$. Corresponding zones on $(B, C)$ plane are shown in Fig.1.

Combining these results with the stability analysis we can distinguish the following zones in the parameter space $(B, C)$ (see Fig.2):

- for negative $E$ : 1) Zone with one unstable regular de Sitter (NOR), 2) zone with one stable (singular) and one unstable (regular) de Sitter $(R O S K)$, 3) zone without de Sitter solutions $(K S M)$.

- for positive E: 1) Zone with one stable regular de Sitter $(P O N), 2)$ zone with one stable (regular) and one unstable (singular) de Sitter (POSL), 3 ) zone without de Sitter solutions ( $L S M)$.

\section{Bianchi I Universe in $3+1$ dimensions}

The situation become less simple even in $3+1$ dimensions if we allow anisotropic perturbations of the de Sitter solution, which means that the Weil tensor has a non-zero contribution to the equations of motion 1 .

Consider a flat homogeneous anisotropic Universe with the Bianchi I metrics in the form

\footnotetext{
${ }^{1}$ Main results of this section have been found independently using another set of variables in 25$]$.
} 


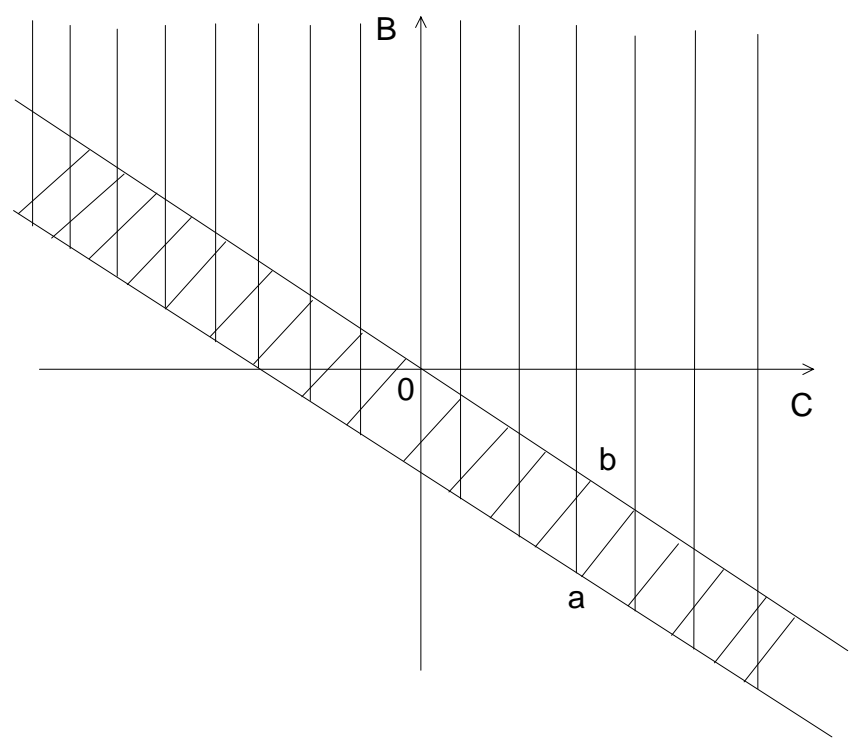

Figure 1: Zones of de Sitter solutions for the action (9). A singular de Sitter exists in double shaded band zone between line a $\left[B+C N>-(N-2)^{2} /(4 \Lambda(N-\right.$ $4))]$ and $\mathbf{b}[B+C N>0]$, a regular de Sitter exists in both double shaded band and shaded zone above the line $\mathbf{b}$.

$$
\mathrm{g}_{i k}=\operatorname{diag}\left(-n(t)^{2}, a(t)^{2}, b(t)^{2}, c(t)^{2}\right) .
$$

It should be noted that in the presence of general quadratic curvature corrections the diagonal form of the metrics (17) is not the general one 24]. We restrict ourself by this special form for simplicity. We show that even in this less general case we have additional restrictions for stability conditions of de Sitter solution. 


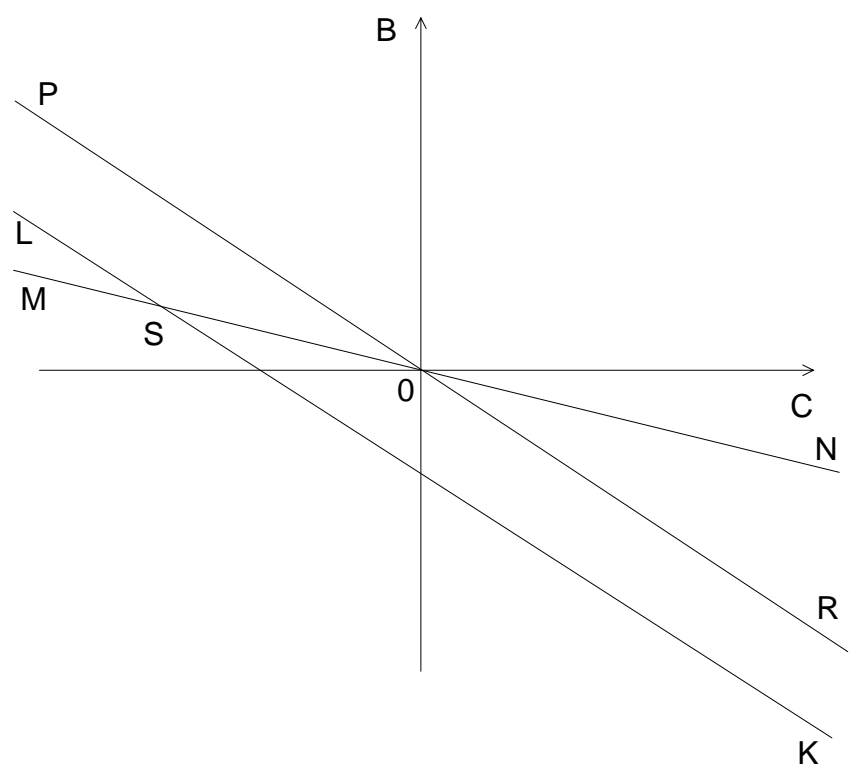

Figure 2: Zones of de Sitter stability for the action (9). The lines $L K$ and $P R$ are the same as in Fig.1; the line $M N$ is the $E=0$ line. The regular de Sitter (if exists) is stable above this line, the singular de Sitter is stable below this line

Starting from the action (3) we have three equations of motion

$$
\begin{aligned}
& 2 \dot{H}_{b}+2 \dot{H}_{c}+2 H_{b}^{2}+2 H_{c}^{2}+2 H_{c} H_{b}-L+2 B\left(H_{b}^{(3)}+3 \dot{H}_{b}^{2}+2 H_{a}^{(3)}+3 \dot{H}_{a}^{2}+H_{a}^{2} \dot{H}_{b}\right. \\
& +3 \ddot{H}_{c} H_{c}+3 \dot{H}_{c}^{2}+H_{c}^{(3)}+H_{b}^{4}-H_{a}^{4}+5 H_{c} \dot{H}_{a} H_{a}+H_{a} H_{c} \dot{H}_{b}-H_{b}^{2} H_{a} H_{c}-H_{c}^{2} H_{a} H_{b} \\
& +H_{b} H_{a}^{2} H_{c}+4 H_{c} \dot{H}_{b} H_{b}+H_{c}^{4}+4 \dot{H}_{c} H_{c}^{2}+4 H_{c} \ddot{H}_{a}+2 H_{c} \ddot{H}_{b}+H_{a} \ddot{H}_{b}+2 H_{b} \ddot{H}_{c} \\
& -H_{c}^{3} H_{a}+H_{c}^{3} H_{b}-H_{a}^{3} H_{c}-H_{a}^{3} H_{b}+H_{b}^{3} H_{c}-H_{b}^{3} H_{a}+2 H_{c}^{2} H_{b}^{2}+H_{a} \ddot{H}_{c}-2 H_{a}^{2} \dot{H}_{a} \\
& +4 H_{a} \ddot{H}_{a}+4 H_{b}^{2} \dot{H}_{b}+3 H_{b} \ddot{H}_{b}+4 H_{b} \ddot{H}_{a}+3 \dot{H}_{a} \dot{H}_{b}+\dot{H}_{a} H_{b}^{2}+3 \dot{H}_{a} \dot{H}_{c}+\dot{H}_{a} H_{c}^{2} \\
& +H_{a}^{2} \dot{H}_{c}+3 \dot{H}_{b} \dot{H}_{c}+2 \dot{H}_{b} H_{c}^{2}+2 H_{b}^{2} \dot{H}_{c}-H_{a} \dot{H}_{b} H_{b}+4 H_{b} \dot{H}_{c} H_{c}-H_{a} \dot{H}_{c} H_{c} \\
& \left.+H_{b} H_{a} \dot{H}_{c}+5 H_{b} \dot{H}_{a} H_{a}+4 H_{c} H_{b} \dot{H}_{a}\right)+4 C\left(2 H_{c}^{(3)}-H_{c}^{2} H_{a}^{2}+6 \ddot{H}_{c} H_{c}+H_{b}^{4}\right. \\
& +5 \dot{H}_{c}^{2}-H_{a}^{4}+H_{c}^{4}+2 H_{a}^{(3)}+3 \dot{H}_{a}^{2}+2 H_{b}^{(3)}+5 \dot{H}_{b}^{2}-2 H_{b} H_{a}^{2} H_{c}+2 H_{c}^{3} H_{b}-2 H_{a}^{3} H_{b} \\
& -2 H_{a}^{3} H_{c}+2 H_{b}^{3} H_{c}-H_{b}^{2} H_{a}^{2}+3 H_{c}^{2} H_{b}^{2}+2 H_{a} \ddot{H}_{c}+2 H_{a} \ddot{H}_{b}+4 H_{c} \ddot{H}_{b}+4 H_{b} \ddot{H}_{a} \\
& +6 H_{b}^{2} \dot{H}_{b}+6 H_{b} \ddot{H}_{b}-2 H_{a}^{2} \dot{H}_{a}+4 H_{a} \ddot{H}_{a}+4 H_{c} \ddot{H}_{a}+6 \dot{H}_{c} H_{c}^{2}+4 \dot{H}_{a} \dot{H}_{b}+2 \dot{H}_{a} H_{b}^{2} \\
& +6 \dot{H}_{b} \dot{H}_{c}+4 \dot{H}_{b} H_{c}^{2}+4 H_{b}^{2} \dot{H}_{c}+4 H_{b} \ddot{H}_{c}+4 \dot{H}_{a} \dot{H}_{c}+2 H_{a} \dot{H}_{c} H_{c}+2 H_{a} \dot{H}_{b} H_{b} \\
& +2 H_{b} H_{a} \dot{H}_{a}+4 H_{c} H_{b} \dot{H}_{a}+2 \dot{H}_{a} H_{c}^{2}+8 H_{c} \dot{H}_{b} H_{b}+2 H_{c} \dot{H}_{a} H_{a}+2 H_{b} H_{a} \dot{H}_{c} \\
& \left.+8 H_{c} H_{b} \dot{H}_{c}+2 H_{c} H_{a} \dot{H}_{b}\right)=0
\end{aligned}
$$


where $H_{a}=\dot{a} / a, H_{b}=\dot{b} / b, H_{c}=\dot{c} / c$, two others can be obtained by transmutation $a \leftrightarrows c$ and $a \leftrightarrows b$

Substituting the de Sitter solution $\left(H_{a}=H_{b}=H_{c}=H_{0}=\right.$ Const), we get that the de Sitter solution has its unperturbed form $H_{0}=\sqrt{\Lambda / 6}$. After evaluating the corresponding system of equation of motion, we find the following eigenvalues

$$
\begin{gathered}
\mu_{1,2,3}=-3 H_{0} \\
\mu_{4,5}=\frac{-18 C H_{0}-6 B H_{0} \pm 2 \sqrt{81 C^{2} H_{0}^{2}+54 B C H_{0}^{2}+9 B^{2} H_{0}^{2}-6 C-2 B}}{2(6 C+2 B)} \\
\mu_{6,7,8,9}=\frac{-3 B H_{0} \pm \sqrt{33 B^{2} H_{0}^{2}+4 B+96 B C H_{0}^{2}}}{2 B} .
\end{gathered}
$$

In expanding Universe we have always $\mu_{1,2,3}<0$. The condition $\mu_{4,5}<0$ leads to the already known result $B+3 C>0$. However, we have now additional restrictions arising from the condition $\mu_{6,7,8,9}<0$. It leads to $\Lambda B+4 C \Lambda+1<0$ if $B>0$ and $\Lambda|B|-4 C \Lambda-1<0$ if $B<0$. Note that these stability conditions now depend not only on coefficients before the higher-order terms in the action (3), but also on the value of the cosmological constant $\Lambda$. These additional restrictions arise from instability with respect to anisotropic perturbations of the de Sitter metrics. It is interesting that independently of $\Lambda$ the whole quadrant $B>0, C>0$, which always satisfies the stability conditions in a FRW Universe, is excluded in anisotropic case (see Fig.3)

In the representation (8) we have the following conditions $4 \tilde{C} \Lambda<\tilde{B} \Lambda / 6-1$ for $\tilde{B}>0$ and $4 \tilde{C} \Lambda>|\tilde{B}| \Lambda / 6-1$ for $\tilde{B}<0$. (see Fig.4).

\section{Conclusions}

We have studied the influence of second order curvature corrections in their general form on stability of de Sitter solution. Our results for the $3+1$ dimensional space-time and for higher-dimensional cases differs significantly. The reason of this difference is non-zero contribution from the Gauss-Bonnet combination for higher-dimensional space-times. In $3+1$ dimension the de Sitter solution always exists, though it may be stable or unstable depending on particular form of the second order corrections. The stability condition takes a very simple and independent of the value of cosmological constant $\Lambda$ form for isotropic Universe, though become more complicated in anisotropic Universe.

In multidimensional Universe even in isotropic case (the only considered in the present paper) there are several possibilities. Depending on the action we may have two de Sitter solutions (in this case one solution is stable and one solution is unstable), one de Sitter solution (which can be either stable or unstable), and there are theories with no de Sitter solutions at all.

There results can be useful in constructions of cosmological scenarios taking into account higher-derivative terms. Instability of a regular de Sitter solutions 


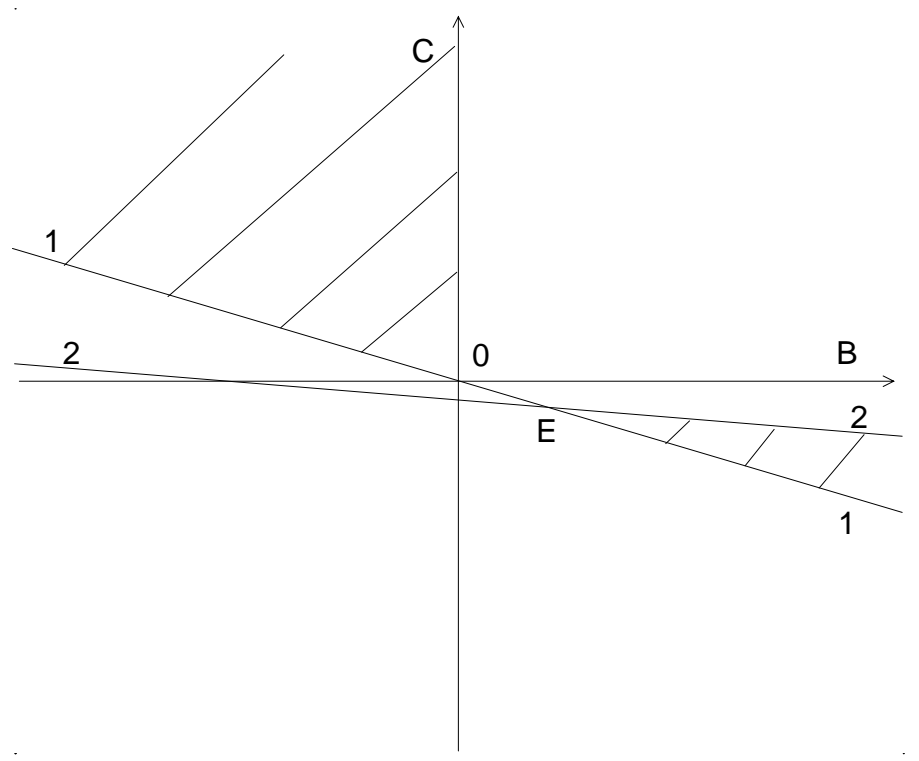

Figure 3: Zones of de Sitter stability for the action (3) (shaded) The de Sitter solutions in zone below the line 1 are unstable on isotropic background, the solutions in the unstable zone above the lines 1 and 2 are unstable due to anisotropic perturbations.

can damage the standard inflationary scenario when close to de Sitter inflationary stage is produced by an effective cosmological constant originated in a matter sector of the theory. This opens a principal possibility to rule out some theories of this type from the observational point of view. On the other hand, unstable singular de Sitter solution can be used in alternative approach to inflation [26]. Our results show that quadratic curvature corrections in Lagrangian can produce such kind of solutions only in multidimensional Universe.

\section{Acknowledgments}

This work is supported by RFBR grant 05-02-17450 and scientific school grant 2338.2003.2 of the Russian Ministry of Science and Technology. Autors are grateful to Alexey Starobinsky, Alan Coley, Sante Carloni, Sigbjorn Hervik and Hans-Jürgen Schmidt for discussions. 


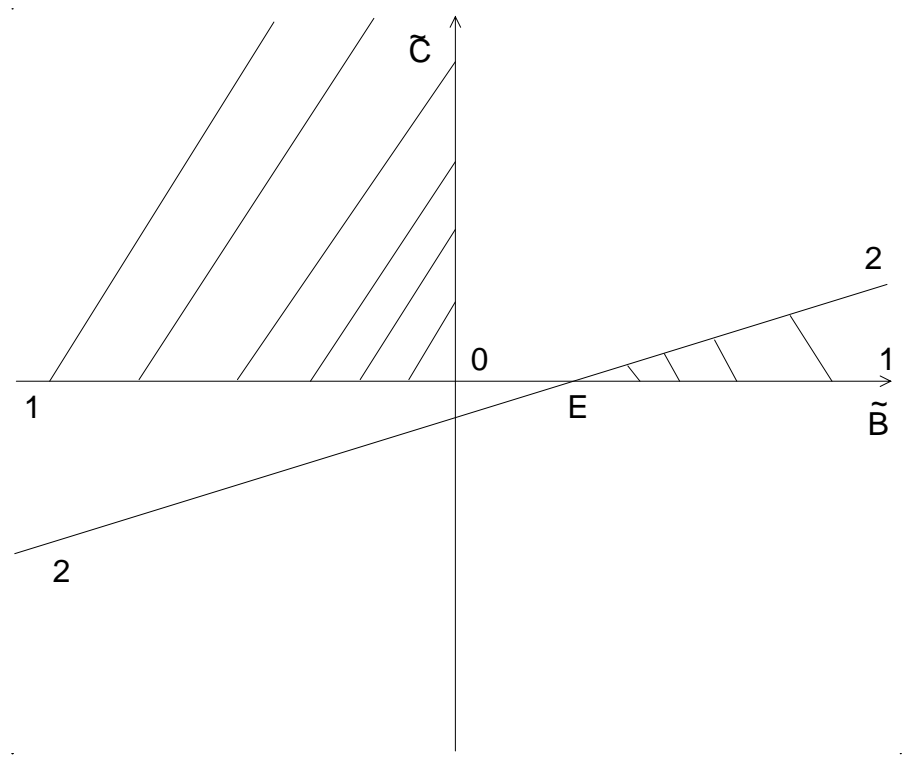

Figure 4: Zones of de Sitter stability for the action (8) (shaded) The unstable zone in the upper half-plane appears due to anisotropic perturbations.

\section{References}

[1] R. Metsaev and A.Tseytlin, Phys. Lett. B185, 52 (1987).

[2] S.Nojiri, S.Odintsov and M.Sami, Phys. Rev. D74 046004 (2006)

[3] G.Cognola, E.Elizade, S.Nojiri, S.Odintsov and S.Zerbini, Phys. Rev. D73 084007 (2006)

[4] D. Lovelock, J. Math. Phys. 12, 498 (1971).

[5] M. Bento and O.Bertolami, Phys. Lett. B368, 198 (1996).

[6] A. V. Smilga, Nucl. Phys. B706, 598 (2005).

[7] M.Pomazanov, V.Kolubasova and S.Alexeyev, "The problem of singularities of higher order curative corrections in four dimensional string gravity", gr-qc/0301029. 
[8] M.Sami, A.Toporensky, P. Tretjakov and S. Tsujikawa, Phys. Lett. B619, 193 (2005).

[9] A. Toporensky and P. Tretyakov, Grav. Cosmol. 12, 55 (2006).

[10] S. Capozziello, S. Carloni and A. Troisi, "Quintessence without scalar field", astro-ph/0303041.

[11] S. Carroll, V. Duvviri, M. Trodden and M.Turner, Phys. Rev. D 70, 043528 (2004).

[12] A. Dolgov and M. Kawasaki, Phys. Lett. b 573, 1 (2003).

[13] V. Faraoni, "Matter instability in modified gravity", astro-ph/0610734.

[14] S.Nojiri and S.Odintsov, Phys. Rev. D 68123512 (2003).

[15] S.Nojiri and S.Odintsov, "Introduction to modified gravity and gravitational alternativ for dark energy", hep-th/0601213.

[16] S.Nojiri and S.Odintsov, "Modified gravity and its reconstruction from the Universe expansion history", hep-th/0611071.

[17] S. Kachru, R. Kallosh, A. Linde and S. P. Trivedi, Phys. Rev. D 68, 046005 (2003).

[18] S. Kachru, R. Kallosh, A. Linde, J. Maldacena, L. McAllister and S. P. Trivedi, JCAP 0310, 013 (2003).

[19] A. Starobinsky and H.-J. Schmidt, Class. Quant. Grav. 4, 695 (1987).

[20] V Müller, H.-J. Schmidt and A. Starobinsky, Phys. Lett. B 202, 198 (1988).

[21] H.-J. Schmidt, Class. Quant. Grav. 5, 233 (1988).

[22] J. Leach, S. Carloni and P. Dunsby, Class. Quant. Grav. 23, 4915 (2006).

[23] S. Carloni, P. Dunsby, S. Capozziello and A. Troisi, Class. Quant. Grav. 22, 4839 (2005).

[24] H.-J. Schmidt, "Lectures on Mathematical Cosmology", gr-qc/0407095.

[25] J.Barrow and S.Hervik, "On the evolution of universes in quadratic theories of gravity", gr-qc/0610013.

[26] A. Starobinsky, Phys. Lett. B91, 99 (1980). 